\title{
RANDOM MOTIONS AT FINITE VELOCITY IN A NON-EUCLIDEAN SPACE
}

\author{
E. ORSINGHER, * University of Rome 'La Sapienza' \\ A. DE GREGORIO, ${ }^{* *}$ University of Padua
}

\begin{abstract}
In this paper telegraph processes on geodesic lines of the Poincaré half-space and Poincaré disk are introduced and the behavior of their hyperbolic distances examined. Explicit distributions of the processes are obtained and the related governing equations derived. By means of the processes on geodesic lines, planar random motions (with independent components) in the Poincaré half-space and disk are defined and their hyperbolic random distances studied. The limiting case of one-dimensional and planar motions together with their hyperbolic distances is discussed with the aim of establishing connections with the well-known stochastic representations of hyperbolic Brownian motion. Extensions of motions with finite velocity to the three-dimensional space are also hinted at, in the final section.
\end{abstract}

Keywords: Hyperbolic distance; telegraph process; Lobachevsky geometry; hyperbolic equation; geodesic line; Bougerol's identity

2000 Mathematics Subject Classification: Primary 60K40

Secondary $53 \mathrm{C} 22$

\section{Introduction}

A type of random motions in non-Euclidean spaces (called hyperbolic Brownian motions) have been examined in some papers appearing in the probabilistic and physical literature.

The first works devoted to diffusions in hyperbolic spaces were published in 1959 in Theory of Probability and its Applications (see Gertsenshtein and Vasiliev (1959) and Karpelevich et al. (1959)), where the propagation of waves in inhomogeneous cables was studied.

Many papers concerning hyperbolic Brownian motion in the upper half-space $\mathrm{H}_{2}^{+}=$ $\{(x, y): y>0, x \in \mathbb{R}\}$ have appeared since (Gruet (1996), (1998), (2000)), also in an applied context (Yor (1992)). Generalizations of hyperbolic Brownian motion in upper spaces $H_{n}^{+}=\left\{\left(x_{1}, \ldots, x_{n}\right): x_{n}>0, x_{j} \in \mathbb{R}, j=1, \ldots, n-1\right\}$ have been studied in the particular case $n=3$ by Karpelevich et al. (1959), Comtet and Monthus (1996), and, for arbitrary values of $n$, Gruet (1996). The branching hyperbolic Brownian motion has also been studied (see Lalley and Sellke (1997)).

The half-plane $\mathrm{H}_{2}^{+}$endowed with the metric

$$
\mathrm{d} s=\frac{\sqrt{\mathrm{d} x^{2}+\mathrm{d} y^{2}}}{y}
$$

Received 20 October 2006; revision received 7 February 2007.

* Postal address: Dipartimento di Statistica, Probabilità e Statistiche Applicate, University of Rome 'La Sapienza', Piazzale Aldo Moro 5, Rome, 00185, Italy. Email address: enzo.orsingher@uniroma1.it

** Current address: Dipartimento di Scienze Economiche, Aziendali e Statistiche, University of Milan, via del Conservatorio 7, Milano, 20122, Italy. 
is a model of hyperbolic spaces introduced by Poincaré. The geodesic curves in $\mathrm{H}_{2}^{+}$are either half-circles whose centers lie on the $x$-axis or vertical half-lines. For an inhomogeneous optical medium where light rays move with speed $c(x, y)=y$ (independent of direction), on the basis of Fermat's principle the possible paths for the light are those curves $L$ which satisfy the equality

$$
\frac{\sin \alpha(y)}{y}=k
$$

where $\alpha(y)$ is the angle between the vertical and the tangent to $L$ at the point with ordinate $y$. It is easy to see that a circle with center on the $x$-axis and radius $1 / k$ satisfies (1.2) (for $k=0$ we get the vertical lines).

In the Poincaré half-plane model the half-circles play the role that straight lines do in the Euclidean space. If the points $A=(x, y)$ and $B=(u, v)$ are such that $x \neq u$, then the hyperbolic distance between $A$ and $B$ is the length of the arc of the circle (with center on the $x$-axis) passing through these points, evaluated by means of the metric (1.1). If $x=u$ then the distance between $A$ and $B$ is also evaluated by means of (1.1) and obviously differs from the usual Euclidean distance (for the geometric properties of $\mathrm{H}_{2}^{+}$, consult Royster (2004) or Terras (1985, Chapter 3, p. 120)).

For the convenience of the reader we sum up some basic results concerning hyperbolic Brownian motion, which is defined as a diffusion on $\mathrm{H}_{2}^{+}$with generator

$$
\frac{y^{2}}{2}\left\{\frac{\partial^{2}}{\partial x^{2}}+\frac{\partial^{2}}{\partial y^{2}}\right\}, \quad y>0 .
$$

Most of the papers dealing with hyperbolic Brownian motion study essentially the behavior of the geodesic distance, $\eta \equiv \eta(t)$, of the moving particle from the origin, $(0,1)$, of $H_{2}^{+}$. Its distribution, denoted by $p_{H_{2}^{+}} \equiv p_{H_{2}^{+}}(\eta, t)$, is obtained by solving the following initial-value problem:

$$
\frac{\partial p}{\partial t}=\frac{1}{2 \sinh \eta} \frac{\partial}{\partial \eta}\left(\sinh \eta \frac{\partial}{\partial \eta}\right) p, \quad \eta>0, t>0, \quad p(\eta, 0)=\delta(\eta),
$$

where $\delta$ is the Dirac function.

In order to reobtain most of the results appearing in the literature, it is better to get rid of the factor $\frac{1}{2}$ in (1.3), by means of the time-scale transformation $t \rightarrow t^{\prime}=t / 2$. The explicit distribution, i.e. $p_{H_{2}^{+}} \equiv p_{H_{2}^{+}}(\eta, t)$, emerging from the solution to (1.3) was obtained first by Gertsenshtein and Vasiliev (1959), without an explicit proof and with some typographical errors.

A detailed derivation of $p_{H_{2}^{+}}$was given in Lao and Orsingher (2007). It takes the form

$$
p_{H_{2}^{+}}(\eta, t)=\frac{\mathrm{e}^{-t / 4}}{\sqrt{\pi}(\sqrt{2 t})^{3}} \int_{\eta}^{\infty} \frac{\varphi \mathrm{e}^{-\varphi^{2} / 4 t}}{\sqrt{\cosh \varphi-\cosh \eta}} \mathrm{d} \varphi,
$$

which coincides with Equation (78) of Monthus and Texier (1996) once the hyperbolic element $\sinh \eta \mathrm{d} \eta$ is considered. In the original time-scale the distribution (1.4) takes the form

$$
p_{H_{2}^{+}}(\eta, t)=\frac{\mathrm{e}^{-t / 8}}{\sqrt{\pi} \sqrt{t^{3}}} \int_{\eta}^{\infty} \frac{\varphi \mathrm{e}^{-\varphi^{2} / 2 t}}{\sqrt{\cosh \varphi-\cosh \eta}} \mathrm{d} \varphi,
$$


and coincides with Equation (b) of Gruet (1996), up to some constants. Some asymptotic results concerning (1.4) were obtained by Simon (2002). On integrating (1.4) and (1.5) we must take into account the hyperbolic element $\sinh \eta \mathrm{d} \eta$. The calculations necessary to check that

$$
\int_{0}^{\infty} p_{H_{2}^{+}}(\eta, t) \sinh \eta \mathrm{d} \eta=1
$$

permit us to identify the exact normalizing constants given (1.4) and (1.5).

The planar hyperbolic Brownian motion also possesses the following stochastic representation, where $B_{1}$ and $B_{2}$ are independent standard Brownian motions:

$$
X(t)=\int_{0}^{t} \mathrm{e}^{B_{1}(s)-s / 2} \mathrm{~d} B_{2}(s), \quad Y(t)=\mathrm{e}^{B_{1}(t)-t / 2} .
$$

In other words, the current coordinates of hyperbolic Brownian motion $X$ and $Y$ satisfy the stochastic differential system

$$
\mathrm{d} X=Y \mathrm{~d} B_{2}, \quad X(0)=0, \quad \mathrm{~d} Y=Y \mathrm{~d} B_{1}, \quad Y(0)=1 .
$$

For the fractional version of (1.3) (without the normalizing constant $\frac{1}{2}$ ), that is,

$$
\frac{\partial^{\alpha} p}{\partial t^{\alpha}}=\frac{1}{\sinh \eta} \frac{\partial}{\partial \eta}\left(\sinh \eta \frac{\partial}{\partial \eta}\right) p, \quad 0<\alpha \leq 1, \quad p(\eta, 0)=\delta(\eta),
$$

we obtain a generalization of (1.4) in the following form:

$$
p_{\alpha}(\eta, t)=\frac{2}{\pi} \int_{0}^{\infty} x E_{\alpha, 1}\left(-\frac{t^{\alpha}}{4}-x^{2} t^{\alpha}\right) \mathrm{d} x \int_{\eta}^{\infty} \frac{\sin x \varphi}{\sqrt{2 \cosh \varphi-2 \cosh \eta}} \mathrm{d} \varphi .
$$

The fractional derivative in (1.7) must be understood in the Dzherbashyan-Caputo sense and $E_{\alpha, 1}(x)$ is the Mittag-Leffler function. The result in (1.8) is particularly interesting when $\alpha=\frac{1}{2}$, because in this case the distribution in (1.8) coincides with that of the hyperbolic Brownian motion where the time is a reflecting Brownian motion $|B(t)|$, that is,

$$
\eta_{1 / 2}=\eta(|B(t)|), \quad t>0 .
$$

In this paper we first consider one-dimensional motions on the geodesic curves of $\mathrm{H}_{2}^{+}$. In particular, we study motions on half-circles orthogonal to the $x$-axis and motions on the positive $y$-axis such that the corresponding hyperbolic distance from $(0,1)$ evaluated by means of (1.1) is equal to $|T(t)|$, where $T$ is the usual symmetric telegraph process. The telegraph process is the simplest one-dimensional motion at finite velocity and many of its properties and distributions have been obtained over the years. Furthermore, it represents a reasonable approximation of Brownian motion and, thus, its hyperbolic version is somehow connected with the so-called stochastic representation of hyperbolic Brownian motion.

If the motion is considered on a vertical half-line (for example the positive $y$-axis), then

$$
Y(t)=\mathrm{e}^{T(t)}, \quad t>0,
$$

has hyperbolic distance from $(0,1)$ given by

$$
\eta(t)=\int_{\min (1, Y(t))}^{\max (1, Y(t))} \frac{\mathrm{d} y}{y}=|T(t)| .
$$


If the finite-velocity random motion develops on the circle of radius 1 and with the origin at $(0,0)$, then the process

$$
\theta(t)=2 \arctan \tanh \frac{U(t)}{2}, \quad-c t \leq U(t) \leq c t,
$$

has hyperbolic distance from $(0,1)$ given by

$$
\eta(t)=\int_{\min (0,2 \arctan \tanh (U(t) / 2))}^{\max (0,2 \arctan \tanh (U(t) / 2))} \frac{\mathrm{d} \theta}{\cos \theta}=|U(t)| .
$$

The hyperbolic distance between the moving points $\left(0, \mathrm{e}^{T(t)}\right)$ and $(\tanh U(t), 1 / \cosh U(t))$ is evaluated by means of

$$
\cosh \eta=\frac{\left(x_{1}-x_{2}\right)^{2}+y_{1}^{2}+y_{2}^{2}}{2 y_{1} y_{2}},
$$

which yields

$$
\cosh \eta=\cosh T(t) \cosh U(t),
$$

the so-called non-Euclidean Pythagorean theorem. Thus, the distance between the moving points on the hyperbolic lines depends on the product of the hyperbolic distances of each point from $(0,1)$. These finite-velocity motions can also be studied in the Poincaré disk obtained from $\mathrm{H}_{2}^{+}$by means of the conformal mapping

$$
w=\frac{z-\mathrm{i}}{-\mathrm{i} z+1} .
$$

The processes in (1.9) and (1.10) can be used to construct two-dimensional motions in $\mathrm{H}_{2}^{+}$ in different manners. The most natural one is perhaps to take

$$
X_{1}(t)=\tanh U(t), \quad Y_{1}(t)=\mathrm{e}^{T(t)},
$$

where $X_{1}(t)$ represents the abscissa of a point moving on the half-circle centered at the origin. This motion has the drawback that it develops only inside the strip $S=\{(x, y): y>0$, $|x| \leq 1\}$.

In the authors' view, however, the most tractable planar motion is described by

$$
X_{2}(t)=\sinh U(t), \quad Y_{2}(t)=\mathrm{e}^{T(t)},
$$

which covers the whole half-space $\mathrm{H}_{2}^{+}$as time goes on. Furthermore, it possesses an interesting limiting relationship with the classical planar hyperbolic Brownian motion. As the parameters $\lambda$ and $c$ tend to $\infty$, the telegraph processes $U$ and $T$ converge weakly to Brownian motions (Kolesnik (2001)). Thus, the vector process (1.12) converges to

$$
X_{2}(t)=\sinh B(t), \quad Y_{2}(t)=\mathrm{e}^{B_{1}(t)},
$$

where $B_{1}$ and $B$ are independent Brownian motions. By Bougerol's identity (see Alili et al. (1997)) we have the following relationship (valid in distribution, i.d.):

$$
\sinh B(t) \stackrel{\text { i.d. }}{=} \int_{0}^{t} \mathrm{e}^{B_{1}(s)} \mathrm{d} B_{2}(s),
$$


which represents the $x$-component of the hyperbolic Brownian motion governed by

$$
\mathrm{d} Y=Y \mathrm{~d} B_{1}+\frac{1}{2} Y \mathrm{~d} t, \quad \mathrm{~d} X=Y \mathrm{~d} B_{2} .
$$

This permits us to state that the planar motion (1.12) is a finite-velocity counterpart of a slightly different version of hyperbolic Brownian motion.

Another alternative definition of a planar motion is given by

$$
X_{3}(t)=\mathrm{e}^{T(t)} \tan \left(2 \arctan \tanh \frac{U(t)}{2}\right)=\mathrm{e}^{T(t)} \sinh U(t), \quad Y_{3}(t)=\mathrm{e}^{T(t)},
$$

which also can move throughout all of $\mathrm{H}_{2}^{+}$, but does not have independent components.

The hyperbolic distances, $\eta_{j}, j=1,2,3$, of the processes in (1.11), (1.12), and (1.14) from $(0,1)$ are given by

$$
\begin{aligned}
\cosh \eta_{1} & =\cosh T(t)+\frac{1}{2} \mathrm{e}^{-T(t)} \tanh ^{2} U(t), \\
\cosh \eta_{2} & =\cosh T(t)+\frac{1}{2} \mathrm{e}^{-T(t)} \sinh ^{2} U(t), \\
\cosh \eta_{3} & =\cosh T(t)+\frac{1}{2} \mathrm{e}^{T(t)} \sinh ^{2} U(t) .
\end{aligned}
$$

We note that

$$
\cosh \eta_{3}-\cosh \eta_{1}=\sinh ^{2} U(t)\left(\sinh T(t)+\frac{1}{2} \mathrm{e}^{-T(t)} \tanh ^{2} U(t)\right),
$$

and that the hyperbolic distance of $\left(X_{3}, Y_{3}\right)$ from the origin is greater than the hyperbolic distance of the first moving particle if $\tanh ^{2} U(t)>1-\mathrm{e}^{2 T(t)}$.

If we compare the hyperbolic distances in (1.15) and (1.16), we find that

$$
\cosh \eta_{3}-\cosh \eta_{2}=\sinh T(t) \sinh ^{2} U(t)
$$

For points of $H_{2}^{+}$such that $0<y<1$, a swift inspection of (1.17) shows that $\eta_{2}>\eta_{3}$. Furthermore, because of the independence and symmetry of $U$ and $T$, we have

$$
\begin{aligned}
\mathrm{E}\left(\cosh \eta_{3}-\cosh \eta_{2}\right) & =0, \\
\mathrm{E}\left(\left(\cosh \eta_{3}-\cosh \eta_{2}\right)^{2}\right) & =\frac{1}{2} \mathrm{E}\left(\mathrm{e}^{2 T}-1\right) \frac{1}{2^{3}} \mathrm{E}\left(\mathrm{e}^{4 U}-4 \mathrm{e}^{2 U}+3\right) .
\end{aligned}
$$

We study the distribution of the hyperbolic distance $\eta_{2}$ and give an explicit formula in terms of the distributions of $U$ and $T$. Simple and explicit expressions are presented for the mean values $\mathrm{E} \cosh \eta_{j}, j=1,2,3$.

The reader must keep in mind that all processes considered here have a double nature. The processes moving with space-varying velocity appear to the Euclidean observer as decelerated or accelerated motions, while the non-Euclidean observer perceives them as movements with constant velocity.

\section{One-dimensional motions with finite velocity in $\mathrm{H}_{2}^{+}$}

The simplest motions at finite velocity which can be studied in $\mathrm{H}_{2}^{+}$are telegraph processes of some type on the geodesic lines. Here we start our analysis with the motions on the positive $y$-axis and on the half-circle with radius 1 and center $(0,0)$. 
Let $T$ be a symmetric telegraph process and let

$$
Y(t)=\mathrm{e}^{T(t)},
$$

which develops on the $y$-axis and starts from $(0,1)$ at $t=0$. The explicit form of $T$ is given by

$$
T(t)=V(0) \int_{0}^{t}(-1)^{N(s)} \mathrm{d} s,
$$

where $V(0)$ is a two-valued random variable (taking the values $\pm c$ with respective probabilities $\frac{1}{2}$ ) independent of $N(t)$, the number of Poisson events in $[0, t]$. The process $T$ depends on the parameters $\lambda$ (the rate of the Poisson process) and $c$ (its speed). The explicit distribution of $T$ is

$$
\begin{aligned}
p_{T}(x, t)= & \frac{\mathrm{e}^{-\lambda t}}{2 c}\left[\lambda I_{0}\left(\frac{\lambda}{c} \sqrt{c^{2} t^{2}-x^{2}}\right)+\frac{\partial}{\partial t} I_{0}\left(\frac{\lambda}{c} \sqrt{c^{2} t^{2}-x^{2}}\right)\right] \mathbf{1}_{\{|x|<c t\}} \\
& +\frac{\mathrm{e}^{-\lambda t}}{2}[\delta(x-c t)+\delta(x+c t)],
\end{aligned}
$$

where $\delta$ is the Dirac function, $I_{0}(x)=\sum_{k=0}^{\infty}(x / 2)^{2 k}\left(1 /(k !)^{2}\right)$ (see Orsingher (1990)), and $\mathbf{1}_{\{\cdot\}}$ is the indicator function.

By applying (1.1) we easily see that the hyperbolic distance of the particle performing the process in (2.1) from the origin, $O \equiv(0,1)$, is

$$
\eta(t)=\int_{\min (1, Y(t))}^{\max (1, Y(t))} \frac{\mathrm{d} y}{y}=|T(t)| .
$$

If the particle approaches the $x$-axis, the Euclidean observer perceives a slowing down in the motion, while the non-Euclidean observer will not notice an analogous phenomenon.

We obtain the mean value of the process in (2.1) in the next theorem.

Theorem 2.1. The mean value of $Y(t), t>0$, is given by

$$
\begin{aligned}
\mathrm{E} Y(t) & =\frac{\mathrm{e}^{-\lambda t}}{2}\left[\left(1+\frac{\lambda}{\sqrt{\lambda^{2}+c^{2}}}\right) \mathrm{e}^{t \sqrt{\lambda^{2}+c^{2}}}+\left(1-\frac{\lambda}{\sqrt{\lambda^{2}+c^{2}}}\right) \mathrm{e}^{-t \sqrt{\lambda^{2}+c^{2}}}\right] \\
& =\mathrm{e}^{-\lambda t}\left[\cosh \left(t \sqrt{\lambda^{2}+c^{2}}\right)+\frac{\lambda}{\sqrt{\lambda^{2}+c^{2}}} \sinh \left(t \sqrt{\lambda^{2}+c^{2}}\right)\right]
\end{aligned}
$$

for all values of $\lambda$ and $c$.

Proof. We first note that, under the condition that $N(t)=n$, from (2.2) the displacement at time $t$ can be written in the form

$$
V(0) \sum_{k=1}^{n+1}(-1)^{k-1}\left(s_{k}-s_{k-1}\right),
$$

where $0=s_{0}<s_{1}<\cdots<s_{k}<\cdots<s_{n}<s_{n+1}=t$ and the instants, $s_{1}, \ldots, s_{n}$, where the Poisson events occur have uniform distribution, that is, its density $g$ reads

$$
g\left(s_{1}, \ldots, s_{n}\right)=\frac{n !}{t^{n}}, \quad 0<s_{1}<\cdots<s_{k}<\cdots<s_{n}<t .
$$


Therefore, we have

$$
\begin{aligned}
\mathrm{E}\left(\mathrm{e}^{T(t)} \mid N(t)=n\right) \\
=\frac{n !}{t^{n}} \int_{0}^{t} \mathrm{~d} s_{1} \cdots \int_{s_{n-1}}^{t} \mathrm{~d} s_{n} \frac{1}{2}\left\{\exp \left(c \sum_{k=1}^{n+1}(-1)^{k}\left(s_{k}-s_{k-1}\right)\right)\right. \\
\left.\quad+\exp \left(-c \sum_{k=1}^{n+1}(-1)^{k}\left(s_{k}-s_{k-1}\right)\right)\right\} \\
=\frac{n !}{t^{n}} \int_{0}^{t} \mathrm{~d} s_{1} \cdots \int_{s_{n-1}}^{t} \mathrm{~d} s_{n} \cosh \left(c \sum_{k=1}^{n+1}(-1)^{k}\left(s_{k}-s_{k-1}\right)\right) \\
=: \frac{n !}{t^{n}} \tau_{n}(t) .
\end{aligned}
$$

We show now that the multiple integrals $\mathcal{I}_{n}(t)$, as a function of time $t$, satisfy the difference differential equation

$$
\frac{\mathrm{d}^{2} \tau_{n}}{\mathrm{~d} t^{2}}=c^{2} \tau_{n}+\tau_{n-2}
$$

where $\mathcal{I}_{0}(t)=\cosh c t$ and $\mathcal{I}_{-1}(t)=0$, by assumption. Equation (2.5) can be obtained by successive differentiations of $\mathcal{I}_{n}(t)$ and by taking into account the fact that the $(n+1)$ th term inside $\tau_{n}(t)$ contains $t$. This means that

$$
\begin{aligned}
\frac{\mathrm{d} \mathcal{I}_{n}}{\mathrm{~d} t}= & \int_{0}^{t} \mathrm{~d} s_{1} \cdots \int_{s_{n-2}}^{t} \mathrm{~d} s_{n-1} \cosh \left(c \sum_{k=1}^{n}(-1)^{k}\left(s_{k}-s_{k-1}\right)\right) \\
& +\int_{0}^{t} \mathrm{~d} s_{1} \cdots \int_{s_{n-1}}^{t} \mathrm{~d} s_{n} \sinh \left(c \sum_{k=1}^{n+1}(-1)^{k}\left(s_{k}-s_{k-1}\right)\right) c(-1)^{n+1} \\
= & I_{n-1}+\int_{0}^{t} \mathrm{~d} s_{1} \cdots \int_{s_{n-1}}^{t} \mathrm{~d} s_{n} \sinh \left(c \sum_{k=1}^{n+1}(-1)^{k}\left(s_{k}-s_{k-1}\right)\right) c(-1)^{n+1} .
\end{aligned}
$$

A further differentiation then gives

$$
\begin{aligned}
\frac{\mathrm{d}^{2} \tau_{n}}{\mathrm{~d} t^{2}}= & \frac{\mathrm{d} \tau_{n-1}}{\mathrm{~d} t}+\int_{0}^{t} \mathrm{~d} s_{1} \cdots \int_{s_{n-2}}^{t} \mathrm{~d} s_{n-1} \sinh \left(c \sum_{k=1}^{n}(-1)^{k}\left(s_{k}-s_{k-1}\right)\right) c(-1)^{n+1} \\
& +\int_{0}^{t} \mathrm{~d} s_{1} \cdots \int_{s_{n-1}}^{t} \mathrm{~d} s_{n} \cosh \left(c \sum_{k=1}^{n+1}(-1)^{k}\left(s_{k}-s_{k-1}\right)\right) c^{2}(-1)^{2(n+1)} \\
= & \frac{\mathrm{d} \tau_{n-1}}{\mathrm{~d} t}+c^{2} \tau_{n}+\left(-\frac{\mathrm{d} \tau_{n-1}}{\mathrm{~d} t}+\tau_{n-2}\right)
\end{aligned}
$$

because, from (2.6), we have

$$
\frac{\mathrm{d} \mathcal{I}_{n-1}}{\mathrm{~d} t}=I_{n-2}+\int_{0}^{t} \mathrm{~d} s_{1} \cdots \int_{s_{n-2}}^{t} \mathrm{~d} s_{n-1} \sinh \left(c \sum_{k=1}^{n}(-1)^{k}\left(s_{k}-s_{k-1}\right)\right) c(-1)^{n} .
$$

This concludes the proof of (2.5). 
If we write

$$
G(t)=\mathrm{E} Y(t)=\sum_{n=0}^{\infty} \mathrm{E}\left(\mathrm{e}^{T(t)} \mid N(t)=n\right) \mathrm{P}(N(t)=n)=\mathrm{e}^{-\lambda t} \sum_{n=0}^{\infty} \lambda^{n} \tau_{n}(t),
$$

then from (2.5) we can extract the differential equation

$$
\sum_{n=0}^{\infty} \lambda^{n} \frac{\mathrm{d}^{2} \tau_{n}}{\mathrm{~d} t^{2}}=c^{2} \sum_{n=0}^{\infty} \lambda^{n} \tau_{n}(t)+\lambda^{2} \sum_{n=2}^{\infty} \lambda^{n-2} \varkappa_{n-2}(t)
$$

whence

$$
\frac{\mathrm{d}^{2}}{\mathrm{~d} t^{2}}\left(\mathrm{e}^{\lambda t} G(t)\right)=c^{2} \mathrm{e}^{\lambda t} G(t)+\lambda^{2} \mathrm{e}^{\lambda t} G(t) .
$$

After some calculations we see that the mean value $G(t)$ is a solution to the second-order linear equation

$$
\frac{\mathrm{d}^{2} G(t)}{\mathrm{d} t^{2}}+2 \lambda \frac{\mathrm{d} G(t)}{\mathrm{d} t}=c^{2} G(t)
$$

The initial conditions are

$$
G(0)=1,\left.\quad \frac{\mathrm{d} G(t)}{\mathrm{d} t}\right|_{t=0}=0 .
$$

While the first condition is straightforward, the second one can be shown by observing that

$$
\frac{\mathrm{d} G(0)}{\mathrm{d} t}=\left.\mathrm{E}\left(\frac{\mathrm{d} T(t)}{\mathrm{d} t} \mathrm{e}^{T(t)}\right)\right|_{t=0}=\mathrm{E}\left(V(0) \mathrm{e}^{T(0)}\right)=\mathrm{E} V(0)=0
$$

because $T(0)=0$ and $V(0)$ is symmetrically distributed. The solution to (2.7) with the initial conditions in (2.8) immediately gives (2.4).

Remark 2.1. In view of the symmetry of the distribution of $T(t)$, we have

$$
\mathrm{Ee}^{-T(t)}=\mathrm{Ee}^{T(t)}
$$

and, thus,

$$
\mathrm{E} \cosh T(t)=\mathrm{Ee}^{T(t)} .
$$

Furthermore, it is obvious from the above calculations that, for all real values of $\beta$,

$$
g(\beta, t):=\mathrm{Ee}^{\beta T(t)}
$$

satisfies

$$
\frac{\partial^{2} g}{\partial t^{2}}+2 \lambda \frac{\partial g}{\partial t}=c^{2} \beta^{2} y
$$

and its explicit form is again as in (2.4) with $c$ replaced by $c \beta$.

The mean value $\mathrm{E} Y(t)$ increases with $c$, because the larger the velocity, the bigger becomes the domain where the particle can be located at time $t$. However, E $Y(t)$ decreases with $\lambda$, because the larger the rate of the Poisson process, the smaller becomes the distance that the particle can run. 
For large values of $t$ we see that

$$
\mathrm{E} Y(t) \sim \frac{1}{2}\left(1+\frac{\lambda}{\sqrt{\lambda^{2}+c^{2}}}\right) \mathrm{e}^{-\lambda t+t \sqrt{\lambda^{2}+c^{2}}} .
$$

It is interesting to note that, as $\lambda, c \rightarrow \infty$ (in such a way that $c^{2} / \lambda \rightarrow 1$ ), the telegraph process converges weakly to Brownian motion (Kolesnik (2001)), and, thus,

$$
\lim _{\lambda, c \rightarrow \infty} \mathrm{E} Y(t)=\mathrm{Ee}^{B(t)}=\mathrm{e}^{t / 2}
$$

as (2.4) confirms after an expansion of $\sqrt{\lambda^{2}+c^{2}}$. In view of all this we note that the normalized process

$$
\hat{Z}(t)=\frac{\mathrm{e}^{T(t)}}{\mathrm{E}^{T(t)}}
$$

converges to the $y$-component of the stochastic representation of the planar hyperbolic Brownian motion in (1.6) (without normalization, it tends to the $y$-component of (1.13)).

Now we study a one-dimensional telegraph process on the half-circle orthogonal to the $x$-axis, depicted in Figure 1 (and denoted by $C_{0}^{+}$for the sake of brevity). We evaluate the hyperbolic distance, $\eta \equiv \eta(t)$, of $P$ from $O$ by applying the metric (1.1), and obtain

$$
\begin{aligned}
\eta(t) & =\int_{O}^{P} \frac{\sqrt{(\mathrm{d} x / \mathrm{d} t)^{2}+(\mathrm{d} y / \mathrm{d} t)^{2}}}{y} \mathrm{~d} t=\int_{0}^{\theta(t)} \frac{\mathrm{d} \theta}{\cos \theta}=\int_{\pi / 2-\theta(t)}^{\pi / 2} \frac{\mathrm{d} \theta}{\sin \theta} \\
& =\left[\log \left|\tan \frac{\theta}{2}\right|\right]_{\pi / 2-\theta(t)}^{\pi / 2}=-\log \left|\tan \left(\frac{\pi}{4}-\frac{\theta(t)}{2}\right)\right| \\
& =-\log \left|\frac{1-\tan (\theta(t) / 2)}{1+\tan (\theta(t) / 2)}\right|=-\log \frac{1-\tan (\theta(t) / 2)}{1+\tan (\theta(t) / 2)}
\end{aligned}
$$

for $\theta(t)>0$ (i.e. $P$ is in the first quadrant). For $\theta(t)<0$ (i.e. $P$ is in the second quadrant) it can easily be seen that

$$
\begin{aligned}
\eta(t) & =\int_{P}^{O} \frac{\sqrt{(\mathrm{d} x / \mathrm{d} t)^{2}+(\mathrm{d} y / \mathrm{d} t)^{2}}}{y} \mathrm{~d} t=-\int_{O}^{P} \frac{\sqrt{(\mathrm{d} x / \mathrm{d} t)^{2}+(\mathrm{d} y / \mathrm{d} t)^{2}}}{y} \mathrm{~d} t \\
& =\log \frac{1-\tan (-|\theta(t)| / 2)}{1+\tan (-|\theta(t)| / 2)}=-\log \frac{1-\tan (|\theta(t)| / 2)}{1+\tan (|\theta(t)| / 2)}
\end{aligned}
$$

If we want $\eta(t)=|U(t)|$, where $U$ is a symmetric telegraph process with parameters $(\lambda, c)$, then from (2.9) we have

$$
\theta(t)=2 \arctan \tanh \frac{U(t)}{2}
$$

Since $\operatorname{arctanh} z=\frac{1}{2} \log [(1+z) /(1-z)]$, from (2.9) and (2.10) we obtain

$$
\eta(t)=2 \operatorname{arctanh} \tan \frac{|\theta(t)|}{2},
$$

and in view of (2.11) it is straightforward that $\eta(t)=|U(t)|$. 


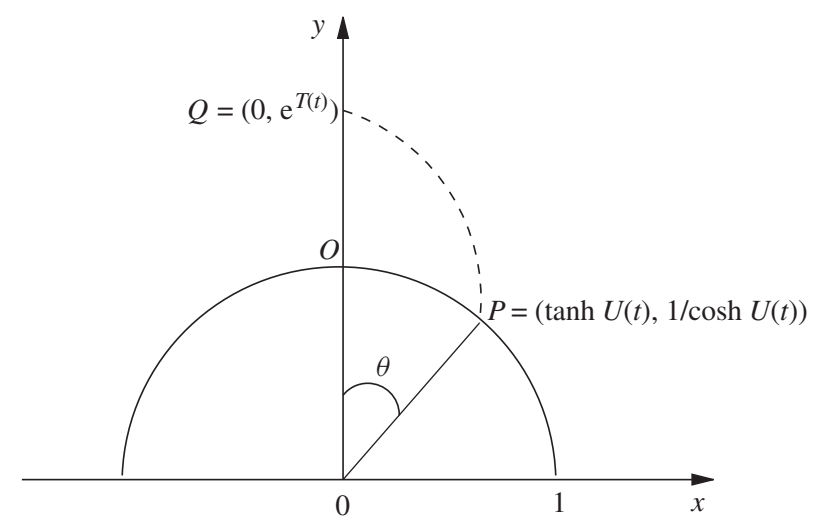

FIGURE 1: Motions at finite velocity on the $y$-axis and on the half-circle $C_{0}^{+}$.

Remark 2.2. The Cartesian coordinates of the moving point on the half-circle $C_{0}^{+}$are $(\tanh U(t), 1 / \cosh U(t))$. The hyperbolic distance between the moving points $\left(0, \mathrm{e}^{T(t)}\right)$ and ( $\tanh U(t), 1 / \cosh U(t))$ is evaluated by means of

$$
\cosh \eta=\frac{\left(x_{1}-x_{2}\right)^{2}+y_{1}^{2}+y_{2}^{2}}{2 y_{1} y_{2}},
$$

again yielding

$$
\cosh \eta=\cosh T(t) \cosh U(t),
$$

the non-Euclidean Pythagorean theorem. Thus, the distance between two points moving on geodesic lines considered above depends from the product of the hyperbolic distances of each of them from the origin. The right triangle $O P Q$ (see Figure 1) has two sides which are chords of half-circles and (2.12) measures the hyperbolic length of its hypotenuse.

We can easily obtain an explicit expression for the mean of (2.12), having in mind (2.4) and performing some calculations. Since $T$ and $U$ are independent, identically distributed random variables, we have

$$
\begin{aligned}
\mathrm{E} \cosh \eta & =\mathrm{E} \cosh T(t) \mathrm{E} \cosh U(t) \\
& =\left\{\frac{\mathrm{e}^{-\lambda t}}{2}\left[\left(1+\frac{\lambda}{\sqrt{\lambda^{2}+c^{2}}}\right) \mathrm{e}^{t \sqrt{\lambda^{2}+c^{2}}}+\left(1-\frac{\lambda}{\sqrt{\lambda^{2}+c^{2}}}\right) \mathrm{e}^{-t \sqrt{\lambda^{2}+c^{2}}}\right]\right\}^{2} \\
& =\mathrm{E} \cosh T(2 t)-\frac{c^{2}}{\lambda^{2}+c^{2}}\left(\mathrm{e}^{-\lambda t} \sinh \left(t \sqrt{\lambda^{2}+c^{2}}\right)\right)^{2} .
\end{aligned}
$$

Remark 2.3. We observe that the formula for the hyperbolic distance of $(x, y)$ from $(0,1)$,

$$
\cosh \eta=\frac{x^{2}+y^{2}+1}{2 y},
$$

can be inferred from the relationships between the hyperbolic coordinates. These formulae read (see Rogers and Williams (1987, p. 213))

$$
x=\frac{\sinh \eta \cos \alpha}{\cosh \eta-\sinh \eta \sin \alpha}, \quad y=\frac{1}{\cosh \eta-\sinh \eta \sin \alpha}
$$




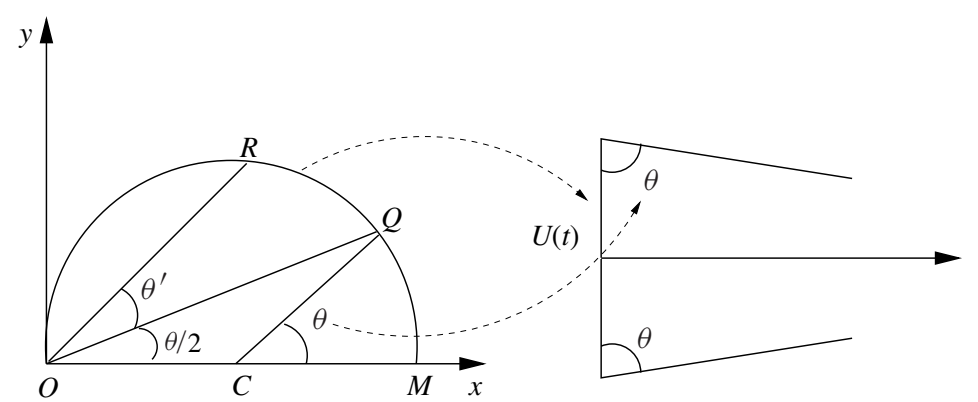

FIGURE 2: Motions on the half-circle and the Lobachevsky angle of parallelism.

and can also be rewritten as

$$
\sinh \eta=\frac{x}{y} \frac{1}{\cos \alpha}, \quad \frac{1}{y}=\cosh \eta-\sinh \eta \sin \alpha .
$$

By $\alpha$ we denote the angle formed by the tangent at $(0,1)$ to the geodesic line passing through $(x, y)$.

From (2.14) we have

$$
\sinh \eta=\frac{x}{y} \frac{1}{\sqrt{1-\left(1 / \sinh ^{2} \eta\right)(\cosh \eta-1 / y)^{2}}}=\frac{x}{y} \frac{\sinh \eta}{\sqrt{-1-1 / y^{2}+2 \cosh \eta / y}} .
$$

In conclusion, $x=\sqrt{-y^{2}-1+2 y \cosh \eta}$, and this leads to (2.13).

Remark 2.4. Another, slightly different, process is obtained by considering the half-circle with center $C$ and radius 1 depicted in Figure 2 . The origin on the circumference is now assumed at the point $R$ (the angle $\widehat{R O M}$ is equal to $\pi / 4$ radians). The moving point $Q$ possesses coordinates $(x=1+\cos \theta, y=\sin \theta)$, and we let $\widehat{Q C M}=\theta$.

The hyperbolic distance, $\eta$, of $Q$ from $R$ (note that $\theta$ decreases when the length of the arc $R Q$ increases) is equal to

$$
\overline{R Q}=\int_{R}^{Q} \frac{\mathrm{d} s}{y}=-\int_{R}^{Q} \frac{\mathrm{d} \theta}{\sin \theta}=\left[-\log \tan \left|\frac{\theta}{2}\right|\right]_{\pi / 2}^{\theta(t)}=-\log \tan \frac{\theta(t)}{2},
$$

for $0<\theta<\pi / 2$. For $\pi / 2<\theta<\pi$, from analogous calculations we have

$$
\overline{R Q}=\int_{Q}^{R} \frac{\mathrm{d} s}{y}=\left[-\log \tan \left|\frac{\theta}{2}\right|\right]_{\theta(t)}^{\pi / 2}=\log \tan \frac{\theta(t)}{2} .
$$

If we want the hyperbolic distance to perform a reflecting telegraph process, then

$$
\theta(t)=2 \arctan \mathrm{e}^{-U(t)},
$$

which is certainly simpler than (2.11). 
Since $\theta^{\prime}=\pi / 4-\theta / 2$ (see Figure 2), the coordinates of $Q$ are $\left(2 \cos ^{2}\left(\pi / 4-\theta^{\prime}\right), \cos 2 \theta^{\prime}\right)=$ $\left(1+\sin 2 \theta^{\prime}, \cos 2 \theta^{\prime}\right)$, and, thus,

$$
\begin{aligned}
\overline{R Q} & =\int_{R}^{Q} \frac{\mathrm{d} s}{y}=\int_{R}^{Q} \frac{2 \mathrm{~d} \theta^{\prime}}{\cos 2 \theta^{\prime}}=\int_{0}^{2 \theta^{\prime}(t)} \frac{\mathrm{d} w}{\cos w}=\int_{\pi / 2-2 \theta^{\prime}(t)}^{\pi / 2} \frac{\mathrm{d} w}{\sin w} \\
& =-\log \left|\tan \left(\frac{\pi}{4}-\theta^{\prime}(t)\right)\right|=-\log \tan \frac{\theta(t)}{2} .
\end{aligned}
$$

This confirms the result in (2.15) for $0<\theta<\pi / 2$. Analogous calculations can be done for the other interval.

We can interpret the process in (2.16) as the angle of parallelism (in the sense of Lobachevsky) between two lines separated by a distance $U(t)$ (randomly changing according to a telegraph process). If we imagine a particle moving on the $y$-axis and we draw the parallel to the $x$-axis at the distance $U(t)$ (see Figure 2), then for $0<\theta<\pi / 2$ we have the right parallel and for $\pi / 2<\theta<\pi$ we have the left parallel, and (2.16) gives the well-known Lobachevsky relationship

$$
\tan \frac{\theta}{2}=\mathrm{e}^{-U(t)}
$$

If $U(t)$ is negative then we have a similar representation in the lower half-plane.

Remark 2.5. From the Lobachevsky relationship (2.17) we have

$$
\mathrm{E} \tan \frac{\theta(t)}{2}=\mathrm{Ee}^{-U(t)}=\mathrm{e}^{-\lambda t}\left[\cosh \left(t \sqrt{\lambda^{2}+c^{2}}\right)+\frac{\lambda}{\sqrt{\lambda^{2}+c^{2}}} \sinh \left(t \sqrt{\lambda^{2}+c^{2}}\right)\right] .
$$

From (2.16) we see that, for $\lambda, c \rightarrow \infty$ in such a way that $c^{2} / \lambda \rightarrow 1, \hat{\theta}$, the limiting process of $\theta$, becomes $\hat{\theta}(t)=2 \arctan \mathrm{e}^{-B(t)}$. Therefore,

$$
\mathrm{E} \tan \frac{\hat{\theta}(t)}{2}=\mathrm{Ee}^{-B(t)}=\mathrm{e}^{t / 2}
$$

which is the limit of (2.18) as $\lambda, c \rightarrow \infty$.

\section{Motion inside the Poincaré disk}

The conformal transformation

$$
w=\frac{z-\mathrm{i}}{-\mathrm{i} z+1}
$$

maps the upper half-plane $H_{2}^{+}$onto the unit-radius disk

$$
D=\left\{(x, y): x^{2}+y^{2}<1\right\}
$$

lying in the complex plane $\mathbb{C}$. In particular, the $x$-axis is mapped by (3.1) onto the border, $\partial D$, of (3.2) and the origin, $(0,1)$, of $H_{2}^{+}$is mapped onto the center of $D$.

The processes dealt with in Section 2, namely

$$
z_{T}=\mathrm{ie}^{T(t)}, \quad z_{U}=\tanh U(t)+\frac{\mathrm{i}}{\cosh U(t)},
$$


are respectively mapped onto

$$
W(t)=\mathrm{i} \tanh \frac{T(t)}{2}, \quad Z(t)=\tanh \frac{U(t)}{2} .
$$

The motion on the half-circle $\mathrm{C}_{0}^{+}$of $\mathrm{H}_{2}^{+}$is transformed by (3.1) into a simple function of the telegraph processes on the $x$-axis of $D$. However, the motion on the $y$-axis maintains its structure. We check that the point $(\tanh U(t), 1 / \cosh U(t))$ is mapped onto the point of $D$ with abscissa $Z(t)$, as follows:

$$
\begin{aligned}
Z(t) & =\frac{\tanh U(t)+\mathrm{i} / \cosh U(t)-\mathrm{i}}{-\mathrm{i}(\tanh U(t)+\mathrm{i} / \cosh U(t))+1} \\
& =\frac{\sinh U(t)+\mathrm{i}(1-\cosh U(t))}{-\mathrm{i} \sinh U(t)+1+\cosh U(t)} \\
& =\frac{2 \sinh (U(t) / 2) \cosh (U(t) / 2)-2 \mathrm{i} \sinh ^{2}(U(t) / 2)}{-2 \mathrm{i} \sinh (U(t) / 2) \cosh (U(t) / 2)+2 \cosh ^{2}(U(t) / 2)} \\
& =\tanh \frac{U(t)}{2}
\end{aligned}
$$

The hyperbolic distance $\eta$ and the Euclidean distance $d$ in $D$ are related by

$$
\eta=\log \frac{1+d}{1-d}
$$

whence

$$
\cosh \eta=\frac{1+d^{2}}{1-d^{2}}
$$

By applying either (3.3) or (3.4) it is easy to prove that the hyperbolic distance of the process $W(t)$ from the origin is equal to $|T(t)|$ and the hyperbolic distance from the center $O$ of $D$ of the process $Z(t)$ is equal to $|U(t)|$.

Remark 3.1. We observe that, since the hyperbolic distance between the points $(x, y)$ and $(0,1)$ of $\mathrm{H}_{2}^{+}$is

$$
\cosh \eta=\frac{x^{2}+y^{2}+1}{2 y}
$$

the distance between their images under the mapping (3.1) does not change. A direct proof of the property of isometry of $(3.1)$ is the following. While $(0,1)$ is mapped onto $O$, the center of $D$, the point $(x, y)$ is mapped onto the point with coordinates

$$
\left(\frac{2 x}{x^{2}+(y+1)^{2}}, \frac{x^{2}+y^{2}-1}{x^{2}+(y+1)^{2}}\right) .
$$

This can be ascertained by writing

$$
w(z)=w(x+\mathrm{i} y)=\frac{x+\mathrm{i} y-\mathrm{i}}{1-\mathrm{i}(x+\mathrm{i} y)}=\frac{x+\mathrm{i}(y-1)}{y+1-\mathrm{i} x}=\frac{2 x+\mathrm{i}\left(x^{2}+y^{2}-1\right)}{x^{2}+(y+1)^{2}} .
$$

In order to check that the hyperbolic distance of the image of $(x, y)$ from $O$ is $\eta$, we perform the following calculations. We start by evaluating $d$, the Euclidean distance of $(x, y)$ from $O$, which is given by

$$
d^{2}=\frac{4 x^{2}+\left(x^{2}+y^{2}-1\right)^{2}}{\left(x^{2}+(y+1)^{2}\right)^{2}} .
$$


From (3.5) we then have

$$
\begin{aligned}
1+d^{2} & =\frac{2 x^{4}+2\left(y^{4}+2 y^{3}+2 y^{2}+2 y+1\right)+4 x^{2}\left(y^{2}+y+1\right)}{\left(x^{2}+(y+1)^{2}\right)^{2}} \\
& =\frac{2\left(x^{2}+y^{2}+1\right)\left(x^{2}+(y+1)^{2}\right)}{\left(x^{2}+(y+1)^{2}\right)^{2}}
\end{aligned}
$$

and

$$
1-d^{2}=\frac{4 y\left(x^{2}+(y+1)^{2}\right)}{\left(x^{2}+(y+1)^{2}\right)^{2}} .
$$

In conclusion,

$$
\cosh \eta=\frac{1+d^{2}}{1-d^{2}}=\frac{x^{2}+y^{2}+1}{2 y}
$$

as claimed.

Remark 3.2. The simple form of the one-dimensional motions on the $x$-and $y$-axes of the Poincaré disk permits us to give distribution of $Z(t)=\tanh U(t)$, which reads

$$
\mathrm{P}(\tanh U(t)<w)=\mathrm{P}(-c t<U(t)<\operatorname{arctanh} w)=\mathrm{P}\left(-c t<U(t)<\frac{1}{2} \log \frac{1+w}{1-w}\right),
$$

where $-1<w<1$. Let $p_{U}(u, t)$ be the absolutely continuous component of the telegraph process $U$ (see (2.3)). Then

$$
p_{Z}(w, t)=\frac{1}{1-w^{2}} p_{U}\left(\frac{1}{2} \log \frac{1+w}{1-w}, t\right)
$$

for $-\tanh c t<w<\tanh c t$.

\section{Planar motions at finite velocity with independent components}

The one-dimensional motions considered in Section 2 provide the tools for constructing planar motions with finite velocity in the hyperbolic plane $\mathrm{H}_{2}^{+}$.

The first possible motion in the plane has independent components given by

$$
X_{1}(t)=\tanh U(t), \quad Y_{1}(t)=\mathrm{e}^{T(t)} .
$$

The hyperbolic distance, $\eta_{1}$, of the moving point from the origin is given by

$$
\cosh \eta_{1}=\cosh T(t)+\frac{1}{2} \mathrm{e}^{-T(t)} \tanh ^{2} U(t) .
$$

Since $\mathrm{E} \cosh T=\mathrm{Ee}^{T}=\mathrm{Ee}^{-T}$, the independence of the processes $T$ and $U$ implies that

$$
\mathrm{E} \cosh \eta_{1}=\mathrm{Ee}^{T(t)}\left\{1+\frac{1}{2} \mathrm{E}_{\tanh ^{2}} U(t)\right\}
$$

An alternative planar motion in $\mathrm{H}_{2}^{+}$is performed by the point with coordinates

$$
X_{2}(t)=\tan \theta(t)=\tan \left(2 \arctan \tanh \frac{U(t)}{2}\right)=\sinh U(t), \quad Y_{2}(t)=\mathrm{e}^{T(t)} .
$$


In this case the hyperbolic distance of the moving point from the origin is given by

$$
\cosh \eta_{2}=\cosh T(t)+\frac{1}{2} \mathrm{e}^{-T(t)} \sinh ^{2} U(t),
$$

and its mean value is

$$
\mathrm{E} \cosh \eta_{2}=\mathrm{Ee}^{T(t)}\left\{1+\frac{1}{2} \mathrm{E} \sinh ^{2} U(t)\right\}=\frac{1}{2^{2}} \mathrm{Ee}^{T(t)}\left\{3+\mathrm{Ee}^{2 U(t)}\right\},
$$

because

$$
\mathrm{E}_{\sinh ^{2}} U(t)=\frac{1}{2}\left(\mathrm{Ee}^{2 U(t)}-1\right)
$$

We note that, as $\lambda, c \rightarrow \infty$ (in such a way that $c^{2} / \lambda \rightarrow 1$ ), $U(t) \stackrel{\mathrm{w}}{\rightarrow} B(t)$ and, thus, the $x$-component in (4.2) converges to sinh $B(t)$. By Bougerol's identity, we can write

$$
\sinh B(t) \stackrel{\text { i.d. }}{=} \int_{0}^{t} \mathrm{e}^{B_{1}(s)} \mathrm{d} B_{2}(s),
$$

showing that the planar process in (4.2) can be viewed as a finite-velocity version of the hyperbolic Brownian motion in (1.13). We also note that, while the motion in (4.1) covers the strip $S=\{(x, y):-1<x<1, y>0\}$, the planar movement in (4.2) can enter all regions of $\mathrm{H}_{2}^{+}$.

Another planar motion the components of which are obtainable from the one-dimensional, finite-velocity processes examined in Section 2 has components

$$
X_{3}(t)=\mathrm{e}^{T(t)} \sinh U(t), \quad Y_{3}(t)=\mathrm{e}^{T(t)} .
$$

In this case we clearly lose the independency of the components of the motion, but maintain the property that the motion is supported all over $H_{2}^{+}$. The hyperbolic distance, $\eta_{3}$, of (4.5) from the origin is given by

$$
\cosh \eta_{3}=\cosh T(t)+\frac{1}{2} \mathrm{e}^{T(t)} \sinh ^{2} U(t),
$$

and has mean value equal to that in (4.4). We also observe that

$$
\cosh \eta_{3}-\cosh \eta_{2}=\sinh T(t) \sinh ^{2} U(t),
$$

and when $T(t)<0$ the moving point is inside the half-circle and the hyperbolic distance $\eta_{3}$ is less than $\eta_{2}$. Of course, if $T(t)>0$ then $\eta_{3}>\eta_{2}$, as a check of the diagram in Figure 3 straightforwardly shows.

The randomly moving point $\left(\tanh U(t), \mathrm{e}^{T(t)}\right)$ is mapped by (3.1) onto the point of $D$ with Cartesian coordinates

$$
\left(\frac{2 \sinh U(t)}{\left(\mathrm{e}^{T(t)}+1\right)^{2}+\sinh ^{2} U(t)}, \frac{\mathrm{e}^{2 T(t)}-1+\sinh ^{2} U(t)}{\left(\mathrm{e}^{T(t)}+1\right)^{2}+\sinh ^{2} U(t)}\right) .
$$

The Euclidean distance, $d$, of this point from the origin is given by

$$
\begin{aligned}
d^{2} & =1-\frac{4 \mathrm{e}^{T(t)}}{\left(\mathrm{e}^{T(t)}+1\right)^{2}+\sinh ^{2} U(t)} \\
& \left.=1-\frac{2}{1+\cosh \eta_{2}} \quad \text { (in view of }(4.3)\right) \\
& =\frac{\cosh \eta_{2}-1}{\cosh \eta_{2}+1} .
\end{aligned}
$$




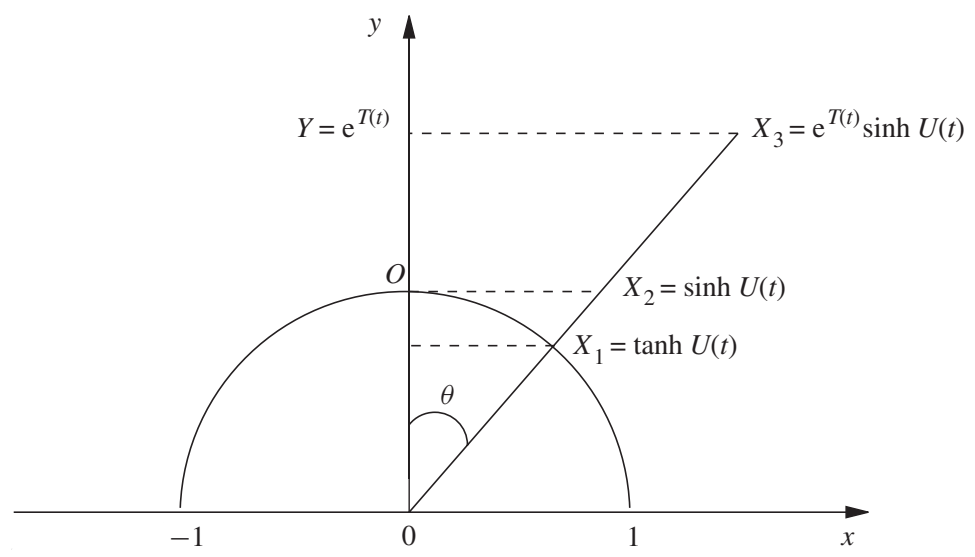

FIGURe 3: Possible definitions of planar motions in the plane.

This is clearly the relationship in (3.4). Furthermore, at each time $t$ the moving particle lies inside a circle with center coinciding with the center of $D$ and radius given by

$$
\begin{aligned}
\max d^{2} & =1-\min \frac{2}{1+\cosh \eta_{2}} \\
& =1-\frac{2}{1+\max \cosh \eta_{2}} \\
& =1-\frac{2}{1+\cosh c t+\frac{1}{2} \mathrm{e}^{c t} \sinh ^{2} c t} .
\end{aligned}
$$

Clearly, $\max d^{2}=0$ for $t=0$ and coincides with the whole of $D$ as $t \rightarrow \infty$.

Similar formulae hold for the other two vector processes.

\section{Governing equations of the probability distributions}

The process $Y=\mathrm{e}^{T(t)}$ developing on the $y$-axis has law $p_{Y}(y, t)$ given by

$$
p_{Y}(y, t)=\frac{1}{y} p_{T}(\log y, t), \quad y>0,
$$

where

$$
\begin{aligned}
p_{T}(\log y, t)= & \frac{\mathrm{e}^{-\lambda t}}{2 c}\left[\lambda I_{0}\left(\frac{\lambda}{c} \sqrt{c^{2} t^{2}-\log ^{2} y}\right)+\frac{\partial}{\partial t} I_{0}\left(\frac{\lambda}{c} \sqrt{c^{2} t^{2}-\log ^{2} y}\right)\right] \mathbf{1}_{\{|\log y|<c t\}} \\
& +\frac{\mathrm{e}^{-\lambda t}}{2}\left[\delta\left(y-\mathrm{e}^{c t}\right)+\delta\left(y-\mathrm{e}^{-c t}\right)\right] .
\end{aligned}
$$

Furthermore, the distribution in (5.1) is the solution to the equation

$$
\frac{\partial^{2} p}{\partial t^{2}}+2 \lambda \frac{\partial p}{\partial t}=c^{2} y \frac{\partial}{\partial y}\left(y \frac{\partial}{\partial y}\right) p, \quad y>0, t>0,
$$

subject to the initial condition

$$
p(y, 0)=\delta(y-1)
$$


This is because the law of the telegraph process solves the telegraph equation

$$
\frac{\partial^{2} p}{\partial t^{2}}+2 \lambda \frac{\partial p}{\partial t}=c^{2} \frac{\partial^{2} p}{\partial x^{2}}
$$

and the transformation $y=\mathrm{e}^{x}$ immediately leads to (5.2).

Equation (5.2) can also be obtained by considering a persistent random walk with velocity depending linearly on the ordinate $y$ of the current position and with changes of direction occurring at Poisson times. Therefore, the joint densities of the distributions

$$
f(y, t) \mathrm{d} y=\mathrm{P}\{Y(t) \in \mathrm{d} y, V(t)=c y\}, \quad b(y, t) \mathrm{d} y=\mathrm{P}\{Y(t) \in \mathrm{d} y, V(t)=-c y\}
$$

satisfy the system of differential equations

$$
\frac{\partial f}{\partial t}=-c y \frac{\partial f}{\partial y}+\lambda(b-f), \quad \frac{\partial b}{\partial t}=c y \frac{\partial b}{\partial y}+\lambda(f-b) .
$$

From (5.3), (5.2) can easily be extracted.

For the process developing on the half-circle $C_{0}^{+}$we have the following result.

Theorem 5.1. The angle process $\theta(t)=2 \arctan \tanh (U(t) / 2)$ has distribution with density

$$
p_{\theta}(\theta, t)=p_{U}\left(-\log \frac{\cos \theta}{1+\sin \theta}, t\right) \frac{1}{\cos \theta}
$$

for $-2 \arctan \tanh (c t / 2)<\theta<2 \arctan \tanh (c t / 2)$, with $p_{U}$ as given by (2.3). Furthermore, the distribution in (5.4) satisfies the partial differential equation

$$
\frac{\partial^{2} p}{\partial t^{2}}+2 \lambda \frac{\partial p}{\partial t}=c^{2} \cos \theta \frac{\partial}{\partial \theta}\left(\cos \theta \frac{\partial}{\partial \theta}\right) p
$$

Proof. We obtain the exact distribution of (2.11) by observing that

$$
\begin{aligned}
\mathrm{P}\left(-2 \arctan \tanh \frac{c t}{2}<\theta(t)<\theta\right) & =\mathrm{P}\left(-2 \arctan \tanh \frac{c t}{2}<2 \arctan \tanh \frac{U(t)}{2}<\theta\right) \\
& =\mathrm{P}\left(-\tanh \frac{c t}{2}<\tanh \frac{U(t)}{2}<\tan \frac{\theta}{2}\right) \\
& =\mathrm{P}\left(\mathrm{e}^{-c t}<\mathrm{e}^{U}<\frac{1+\tan (\theta / 2)}{1-\tan (\theta / 2)}\right) \\
& =\mathrm{P}\left(-c t<U<-\log \frac{1-\tan (\theta / 2)}{1+\tan (\theta / 2)}\right) .
\end{aligned}
$$

In the above steps we have used the facts that the function $\theta(t)$ defined in (2.11) varies in $(-\pi / 2, \pi / 2), \tanh x$ is a monotone increasing function, and the inverse of $\tanh x$ is also increasing. Hence, we have

$$
\begin{aligned}
p_{\theta}(\theta, t)=\frac{\mathrm{d}}{\mathrm{d} \theta} \mathrm{P}(\theta(t)<\theta) & =p_{U}\left(-\log \frac{1-\tan (\theta / 2)}{1+\tan (\theta / 2)}, t\right) \frac{1}{\cos \theta} \\
& =p_{U}\left(-\log \frac{\cos \theta}{1+\sin \theta}, t\right) \frac{1}{\cos \theta}
\end{aligned}
$$


for

$$
-2 \arctan \tanh \left(\frac{c t}{2}\right)<\theta<2 \arctan \tanh \left(\frac{c t}{2}\right) .
$$

We can also obtain explicitly the partial differential equation satisfied by the distribution in (5.6). By (2.11) we must perform the transformation

$$
\theta=2 \arctan \tanh \frac{u}{2}
$$

in the telegraph equation. We find that

$$
\frac{\partial}{\partial u}=\frac{\partial}{\partial \theta} \frac{1}{1+\tanh ^{2}(u / 2)} \frac{1}{\cosh ^{2}(u / 2)}=\frac{\partial}{\partial \theta} \frac{1}{\cosh u}=\frac{\partial}{\partial \theta} \cos \theta,
$$

taking into account the fact that

$$
\tan \theta=\frac{2 \tanh (u / 2)}{1-\tanh ^{2}(u / 2)}=\sinh u
$$

and, thus, $\cosh u=\sqrt{1+\sinh ^{2} u}=\sqrt{1+\tan ^{2} \theta}=1 / \cos \theta$. Therefore, in this case the governing equation is

$$
\frac{\partial^{2} p}{\partial t^{2}}+2 \lambda \frac{\partial p}{\partial t}=c^{2} \cos \theta \frac{\partial}{\partial \theta}\left(\cos \theta \frac{\partial}{\partial \theta}\right) p .
$$

Telegraph-type equations with space-varying velocities, like (5.2) and (5.5), have been studied by Ratanov (1999). Models of this type can be fruitfully applied in the study of motions in inhomogeneous media, where the velocity of particles is locally determined by the irregular structure of the material.

\section{On the behavior of the hyperbolic distance in $\mathrm{H}_{2}^{+}$for motions with finite velocity}

We here consider a planar motion in $\mathrm{H}_{2}^{+}$and focus our attention on the behavior of the hyperbolic distance of the randomly moving particle from the origin. The problem of finding the exact distribution of the hyperbolic distance of the moving point from the origin of $\mathrm{H}_{2}^{+}$ is complicated by the awkward expressions for $\cosh \eta_{j}, j=1,2,3$. The simplest hyperbolic distance is that in (4.3). We give an expression for it in the next theorem.

Theorem 6.1. The hyperbolic distance from the origin, $\eta_{2}$, of the planar process with components $\left(\sinh U(t), \mathrm{e}^{T(t)}\right)$ has distribution given by

$$
\begin{aligned}
\mathrm{P}\left(1 \leq \cosh \eta_{2} \leq z\right)=\int_{\max \left(-c t,-\log \left(z+\sqrt{z^{2}-1}\right)\right)}^{\min \left(c t, \log \left(z+\sqrt{z^{2}-1}\right)\right)} & \mathrm{P}\left(\cosh v \leq \cosh \eta_{2} \leq z \mid T(t)=v\right) \\
& \times \mathrm{P}(T(t) \in \mathrm{d} v)
\end{aligned}
$$

with density

$$
\begin{aligned}
\frac{\mathrm{d}}{\mathrm{d} z} \mathrm{P}\left(1 \leq \cosh \eta_{2} \leq z\right)= & \int_{\max \left(-c t,-\log \left(z+\sqrt{z^{2}-1}\right)\right.}^{\min \left(c t, \log \left(z+\sqrt{z^{2}-1}\right)\right)} \frac{\sqrt{2} \mathrm{e}^{v / 2}}{\sqrt{z-\cosh v} \sqrt{1+2 \mathrm{e}^{v}(z-\cosh v)}} \\
& \times p_{U}\left(\operatorname{arcsinh}\left(\sqrt{2} \mathrm{e}^{v / 2} \sqrt{z-\cosh v}\right), t\right) \mathrm{P}(T(t) \in \mathrm{d} v),
\end{aligned}
$$

for $1 \leq z \leq \cosh c t+\left(\mathrm{e}^{c t} / 2\right) \sinh ^{2} c t$, where $p_{U}(z, t) \mathrm{d} z=\mathrm{P}(U(t) \in \mathrm{d} z)$ is as given in $(2.3)$. 
Proof. We first give the following conditional distribution:

$$
\begin{aligned}
\mathrm{P}\left(\cosh v \leq \cosh \eta_{2} \leq z \mid T(t)=v\right) \\
=\mathrm{P}\left(\cosh v \leq \cosh T(t)+\frac{1}{2} \mathrm{e}^{-T(t)} \sinh ^{2} U(t) \leq z \mid T(t)=v\right) \\
=2 \mathrm{P}\left(0 \leq U(t) \leq \operatorname{arcsinh}\left(\sqrt{2}(z-\cosh T(t))^{1 / 2} \mathrm{e}^{T(t) / 2}\right) \mid T(t)=v\right) \\
=2 \mathrm{P}\left(0 \leq U(t) \leq \log \left(\sqrt{2} \mathrm{e}^{v / 2}(z-\cosh v)^{1 / 2}\right.\right. \\
\left.\left.\quad+\left(1+2 \mathrm{e}^{v}(z-\cosh v)\right)^{1 / 2}\right) \mid T(t)=v\right) .
\end{aligned}
$$

The conditional probability (6.2) is defined on the interval

$$
K=\left\{z: \cosh v \leq z \leq \cosh v+\frac{1}{2} \mathrm{e}^{-v} \sinh ^{2} c t\right\},
$$

as can be ascertained from (4.3). It is easy to check that

$$
\begin{aligned}
& \mathrm{P}\left(\cosh v \leq \cosh \eta_{2} \leq \cosh v+\frac{1}{2} \mathrm{e}^{-v} \sinh ^{2} c t \mid T(t)=v\right) \\
& =2 \mathrm{P}\left(0 \leq U(t) \leq \log \left(\sinh c t+\sqrt{1+\sinh ^{2} c t}\right)\right) \\
& =2 \mathrm{P}(0 \leq U(t) \leq c t) \\
& =2 \int_{0}^{c t} p_{U}(u, t) \mathrm{d} u \\
& =1
\end{aligned}
$$

The bounds in the term on the right-hand side of the first equality in (6.3) are obtained by taking $z$ equal to the right and left limits of $K$.

We can also extract from (6.2) the explicit form of the density of $\cosh \eta_{2}$, as follows:

$$
\begin{aligned}
& \frac{\mathrm{d}}{\mathrm{d} z} \mathrm{P}\left(\cosh v \leq \cosh \eta_{2} \leq z \mid T(t)=v\right) \\
& \quad=\frac{\sqrt{2} \mathrm{e}^{v / 2}}{\sqrt{z-\cosh v} \sqrt{1+2 \mathrm{e}^{v}(z-\cosh v)}} p_{U}\left(\operatorname{arcsinh}\left(\sqrt{2}(z-\cosh v)^{1 / 2} \mathrm{e}^{v / 2}\right), t\right)
\end{aligned}
$$

for $\cosh v \leq z \leq \cosh v+\frac{1}{2} \mathrm{e}^{-v} \sinh ^{2} c t$. Here $p_{U} \equiv p_{U}(u, t)$ is the density of the absolutely continuous component of the telegraph process. The distribution of $\cosh \eta_{2}$ can be inferred from (6.2) and takes the following form:

$$
\begin{aligned}
\mathrm{P}(1 \leq & \left.\cosh \eta_{2} \leq z\right) \\
& =\int_{\max \left(-c t,-\log \left(z+\sqrt{z^{2}-1}\right)\right)}^{\min \left(c t, \log \left(z+\sqrt{z^{2}-1}\right)\right)} \mathrm{P}\left(\cosh v \leq \cosh \eta_{2} \leq z \mid T(t)=v\right) \mathrm{P}(T(t) \in \mathrm{d} v) .
\end{aligned}
$$

The interval where the distribution (6.1) is defined is

$$
J=\left\{1, \cosh c t+\frac{\mathrm{e}^{c t}}{2} \sinh ^{2} c t\right\} .
$$

The upper bound of $J$ is obtained by observing that

$$
\max \cosh \eta_{2}=\max \cosh T(t)+\frac{1}{2} \mathrm{e}^{-\min T(t)} \max \sinh ^{2} U(t) .
$$


From (6.1) we can infer the explicit density of the distribution of the hyperbolic distance $\cosh \eta_{2}(t)$. Since

$$
\mathrm{P}\left(\cosh v \leq \cosh \eta_{2} \leq z \mid T(t)=v\right)=0
$$

for $v= \pm \log \left(z+\sqrt{z^{2}-1}\right)$, we have

$$
\begin{aligned}
\frac{\mathrm{d}}{\mathrm{d} z} \mathrm{P}(1 & \left.\leq \cosh \eta_{2} \leq z\right) \\
=\int_{\max \left(-c t,-\log \left(z+\sqrt{z^{2}-1}\right)\right)}^{\min \left(c t, \log \left(z+\sqrt{z^{2}-1}\right)\right)} & \frac{\sqrt{2} \mathrm{e}^{v / 2}}{\sqrt{z-\cosh v} \sqrt{1+2 \mathrm{e}^{v}(z-\cosh v)}} \\
& \times p_{U}\left(\operatorname{arcsinh}\left(\sqrt{2} \mathrm{e}^{v / 2} \sqrt{z-\cosh v}\right), t\right) \mathrm{P}\{T(t) \in \mathrm{d} v\}
\end{aligned}
$$

for $z \in J$.

Remark 6.1. In the limiting case (where $\lambda, c \rightarrow \infty$ in such a way that $c^{2} / \lambda \rightarrow 1$ ), the planar motion in (4.2) tends to a version of the hyperbolic Brownian motion with components $\left(\mathrm{e}^{B_{1}(t)}, \sinh B_{2}(t)\right)$, the hyperbolic distance of which is clearly given by

$$
\cosh \bar{\eta}_{2}=\cosh B_{1}(t)+\frac{\mathrm{e}^{-B_{1}(t)}}{2} \sinh ^{2} B_{2}(t) .
$$

In (6.2) and (6.1) the distributions of the telegraph processes must be replaced by those of Brownian motions $B_{1}$ and $B_{2}$ and the intervals $K$ and $J$ must undergo the necessary modifications.

Remark 6.2. A different approach to the problem of studying the evolution of the hyperbolic distance $\eta \equiv \eta(t)$ of planar motions consists in describing $\eta(t)$ as a telegraph process with space-dependent speed $v(z)=c / z$, where $c$ is some positive constant. This represents a sort of qualitative approximation to the process described by $\eta$. Near the origin, the particle is pushed away at high velocity and slows down if its distance from the origin increases. A Poisson process governs the durations of the periods in which $\eta$ increases and decreases.

If we assume that the direction of motion is reversed at Poisson times, then the probability functions

$$
f(z, t) \mathrm{d} z=\mathrm{P}\left(X(t) \in \mathrm{d} z, V(t)=\frac{c}{z}\right), \quad b(z, t) \mathrm{d} z=\mathrm{P}\left(X(t) \in \mathrm{d} z, V(t)=-\frac{c}{z}\right),
$$

related to the particle position $X(t)$ and the current speed $V(t)$, satisfy the system of differential equations

$$
\begin{aligned}
& \frac{\partial f}{\partial t}=-\frac{c}{z} \frac{\partial f}{\partial z}+\lambda(b-f), \\
& \frac{\partial b}{\partial t}=\frac{c}{z} \frac{\partial b}{\partial z}+\lambda(f-b) .
\end{aligned}
$$

The reasoning leading to (6.4) is that the position $z$ is reached at time $t+\mathrm{d} t$ if at time $t$ the particle starts at $z-\mathrm{d} z^{\prime}$ with speed $c /\left(z-\mathrm{d} z^{\prime}\right)$, where the displacement $\mathrm{d} z^{\prime}$ must be such that

$$
z-\mathrm{d} z^{\prime}+\frac{c}{z-\mathrm{d} z^{\prime}} \mathrm{d} t=z
$$

so that $\mathrm{d} z^{\prime}=c \mathrm{~d} t / z$ (up to negligible infinitesimals). 
From (6.4) and (6.5) it is easy to show that $p=f+b$ satisfies the partial differential equation

$$
\frac{\partial^{2} p}{\partial t^{2}}+2 \lambda \frac{\partial p}{\partial t}=\frac{c^{2}}{z} \frac{\partial}{\partial z}\left(\frac{1}{z} \frac{\partial}{\partial z}\right) p
$$

By means of the transformation $z=\sinh \eta$, where $\eta$ is the hyperbolic distance of a point $(x, y)$ of $\mathrm{H}_{2}^{+}$from the origin, $(0,1)$, we extract from (6.6) the following equation, where $2 \eta=\eta^{\prime}$ :

$$
\begin{aligned}
\frac{\partial^{2} p}{\partial t^{2}}+2 \lambda \frac{\partial p}{\partial t} & =\frac{c^{2}}{\sinh \eta \cosh \eta} \frac{\partial}{\partial \eta}\left(\frac{1}{\sinh \eta \cosh \eta} \frac{\partial}{\partial \eta}\right) p \\
& =\frac{(2 c)^{2}}{\sinh (2 \eta)} \frac{\partial}{\partial \eta}\left(\frac{1}{\sinh (2 \eta)} \frac{\partial}{\partial \eta}\right) p \\
& =\frac{2^{4} c^{2}}{\sinh \eta^{\prime}} \frac{\partial}{\partial \eta^{\prime}}\left(\frac{1}{\sinh \eta^{\prime}} \frac{\partial}{\partial \eta^{\prime}}\right) p .
\end{aligned}
$$

Equation (6.7) is therefore related to a motion where the hyperbolic distance from the origin varies with speed $c / \sinh \eta$, decreasing with $\eta$.

In the case of the Kac limit $\left(\lambda, c \rightarrow \infty\right.$ in such a way that $\left.c^{2} / \lambda \rightarrow 1\right)$, from (6.7) we obtain the heat equation in the form

$$
\begin{aligned}
\frac{\partial p}{\partial t} & =\frac{2^{3}}{\sinh \eta^{\prime}} \frac{\partial}{\partial \eta^{\prime}}\left(\frac{1}{\sinh \eta^{\prime}} \frac{\partial}{\partial \eta^{\prime}}\right) p \\
& =\frac{2^{3}}{\sinh \eta^{\prime}}\left\{\frac{\partial}{\partial \eta^{\prime}}\left(\sinh \eta^{\prime} \frac{\partial}{\partial \eta^{\prime}}\right)+\frac{\partial}{\partial \eta^{\prime}}\left(\frac{1-\sinh ^{2} \eta^{\prime}}{\sinh \eta^{\prime}} \frac{\partial}{\partial \eta^{\prime}}\right)\right\} p .
\end{aligned}
$$

For $\sinh \eta^{\prime} \sim 1$, that is, for $\eta^{\prime} \sim \log (1+\sqrt{2})$, the second term in (6.8) is negligible. In the neighborhood of $\eta^{\prime}=\log (1+\sqrt{2})$, the solution of $(6.7)$, subject to $p\left(\eta^{\prime}, 0\right)=\delta\left(\eta^{\prime}\right)$, is similar to that of classical hyperbolic Brownian motion.

\section{Some remarks concerning hyperbolic Brownian motion in the space $\mathrm{H}_{3}^{+}$}

A possible three-dimensional motion with finite velocity can be obtained by generalizing ideas of Section 4. We can define the process

$$
X(t)=\sinh U(t), \quad Y(t)=\sinh V(t), \quad Z(t)=\mathrm{e}^{T(t)} .
$$

The processes $T, U$, and $V$ are independent telegraph processes. The idea underlying the definition in (7.1) is to consider half-circle geodesics in the upper half-planes $\{(x, z): x \in \mathbb{R}$, $z>0\}$ and $\{(y, z): y \in \mathbb{R}, z>0\}$ and processes similar to (2.11) on each of them. The $X$ and $Y$ components of three-dimensional motion are then defined as in Section 3. This procedure can be generalized to the space $H_{n}^{+}$.

As $\lambda, c \rightarrow \infty$ with $c^{2} / \lambda \rightarrow 1$, the vector process in (7.1) converges to

$$
\widetilde{X}(t)=\sinh B_{1}(t), \quad \tilde{Y}(t)=\sinh B_{2}(t), \quad \widetilde{Z}(t)=\mathrm{e}^{B_{3}(t)},
$$

where $B_{1}, B_{2}$, and $B_{3}$ are independent Brownian motions. By Bougerol's identity, the vector process in (7.2) is equal in distribution to

$$
X(t)=\int_{0}^{t} \mathrm{e}^{B_{3}(s)} \mathrm{d} B_{1}(s), \quad Y(t)=\int_{0}^{t} \mathrm{e}^{B_{3}(s)} \mathrm{d} B_{2}(s), \quad Z(t)=\mathrm{e}^{B_{3}(t)} .
$$


Clearly, the vector process in (7.3) is the solution to the system of stochastic differential equations

$$
\mathrm{d} X=Z \mathrm{~d} B_{1}, \quad \mathrm{~d} Y=Z \mathrm{~d} B_{2}, \quad \mathrm{~d} Z=Z \mathrm{~d} B_{3}+\frac{1}{2} Z \mathrm{~d} t
$$

with initial conditions $X(0)=Y(0)=0$ and $Z(0)=1$.

Recall that the hyperbolic Brownian motion in $H_{3}^{+}=\left\{(x, y, z): z>0,(x, y) \in \mathbb{R}^{2}\right\}$ is a diffusion with generator

$$
\frac{z^{2}}{2}\left\{\frac{\partial^{2}}{\partial x^{2}}+\frac{\partial^{2}}{\partial y^{2}}+\frac{\partial^{2}}{\partial z^{2}}\right\}
$$

It possesses the simple stochastic representation (see Gruet (1996))

$$
X(t)=\int_{0}^{t} \mathrm{e}^{B_{1}(s)-s / 2} \mathrm{~d} B_{2}(s), \quad Y(t)=\int_{0}^{t} \mathrm{e}^{B_{1}(s)-s / 2} \mathrm{~d} B_{3}(s), \quad Z(t)=\mathrm{e}^{B_{1}(t)-t / 2},
$$

where $B_{1}, B_{2}$, and $B_{3}$ are independent Brownian motions.

Theorem 7.1. The hyperbolic distance $\eta(t), t>0$, of hyperbolic Brownian motion from the origin, $(0,0,1)$, of $\mathrm{H}_{3}^{+}$has distribution

$$
p_{H_{3}^{+}}(\eta, t)=\frac{\mathrm{e}^{-t}}{2 \sqrt{\pi} t^{3 / 2}} \frac{\eta \mathrm{e}^{-\eta^{2} / 4 t}}{\sinh \eta}, \quad \eta>0,
$$

and is the solution to the initial-value problem

$$
\frac{\partial p}{\partial t}=\frac{1}{\sinh ^{2} \eta} \frac{\partial}{\partial \eta}\left(\sinh ^{2} \eta \frac{\partial}{\partial \eta}\right) p, \quad p(\eta, 0)=\delta(\eta) .
$$

Proof. We show that (7.4) satisfies (7.5) directly. Let us write

$$
g(\eta, t)=\frac{\mathrm{e}^{-t} \eta \mathrm{e}^{-\eta^{2} / 4 t}}{t^{3 / 2} \sinh \eta}
$$

Some calculations show that

$$
\begin{gathered}
\frac{\partial g}{\partial t}=\frac{\mathrm{e}^{-t} t^{-3 / 2} \eta \mathrm{e}^{-\eta^{2} / 4 t}}{\sinh \eta}\left\{-1-\frac{3}{2 t}+\frac{\eta^{2}}{4 t^{2}}\right\}, \\
\frac{\partial g}{\partial \eta}=\frac{\mathrm{e}^{-t} t^{-3 / 2} \mathrm{e}^{-\eta^{2} / 4 t}}{\sinh \eta}\left\{1-\eta \frac{\cosh \eta}{\sinh \eta}-\frac{\eta^{2}}{2 t}\right\}, \\
\left(\sinh ^{2} \eta \frac{\partial}{\partial \eta}\right) g=\mathrm{e}^{-t} t^{-3 / 2} \mathrm{e}^{-\eta^{2} / 4 t}\left\{\sinh \eta-\eta \cosh \eta-\frac{\eta^{2}}{2 t} \sinh \eta\right\}, \\
\frac{\partial}{\partial \eta}\left(\sinh ^{2} \eta \frac{\partial}{\partial \eta}\right) g=\mathrm{e}^{-t} t^{-3 / 2} \mathrm{e}^{-\eta^{2} / 4 t} \eta \sinh \eta\left\{-1-\frac{3}{2 t}+\frac{\eta^{2}}{4 t^{2}}\right\} .
\end{gathered}
$$

From this we easily find that

$$
\frac{1}{\sinh ^{2} \eta} \frac{\partial}{\partial \eta}\left(\sinh ^{2} \eta \frac{\partial}{\partial \eta}\right) g=\mathrm{e}^{-t} t^{-3 / 2} \mathrm{e}^{-\eta^{2} / 4 t} \frac{\eta}{\sinh \eta}\left\{-1-\frac{3}{2 t}+\frac{\eta^{2}}{4 t^{2}}\right\},
$$


which coincides with (7.6), thus proving that the function $g$ is a solution to the equation

$$
\frac{\partial p}{\partial t}=\frac{1}{\sinh ^{2} \eta} \frac{\partial}{\partial \eta}\left(\sinh ^{2} \eta \frac{\partial}{\partial \eta}\right) p .
$$

The value of the normalizing constant, $C=1 / 2 \sqrt{\pi}$, in (7.4) is evaluated below (and slightly differs from that appearing in Karpelevich et al. (1959)). Taking into account the hyperbolic element $\sinh ^{2} \eta \mathrm{d} \eta$, we have

$$
\begin{aligned}
1 & =C \mathrm{e}^{-t} t^{-3 / 2} \int_{0}^{\infty} \eta \mathrm{e}^{-\eta^{2} / 4 t} \sinh \eta \mathrm{d} \eta \\
& =C \frac{\mathrm{e}^{-t}}{2} t^{-3 / 2} \int_{0}^{\infty} \eta \mathrm{e}^{-\eta^{2} / 4 t}\left(\mathrm{e}^{\eta}-\mathrm{e}^{-\eta}\right) \mathrm{d} \eta \\
& =C \frac{1}{2} t^{-3 / 2} \int_{0}^{\infty} \eta\left(\mathrm{e}^{-(\eta-2 t)^{2} / 4 t}-\mathrm{e}^{-(\eta+2 t)^{2} / 4 t}\right) \mathrm{d} \eta \\
& =C \frac{1}{2} t^{-3 / 2} \sqrt{2 t}\left\{\int_{-\sqrt{2 t}}^{\infty}(2 t+w \sqrt{2 t}) \mathrm{e}^{-w^{2} / 2} \mathrm{~d} w-\int_{\sqrt{2 t}}^{\infty}(w \sqrt{2 t}-2 t) \mathrm{e}^{-w^{2} / 2} \mathrm{~d} w\right\} \\
& =C \frac{1}{2} t^{-3 / 2} \sqrt{2 t} 2 t \int_{-\infty}^{\infty} \mathrm{e}^{-w^{2} / 2} \mathrm{~d} w \\
& =2 \sqrt{\pi} C .
\end{aligned}
$$

Therefore, the explicit expression for $p_{H_{3}^{+}}(\eta, t)$ is

$$
p_{H_{3}^{+}}(\eta, t)=\frac{1}{2 \sqrt{\pi}} g(\eta, t),
$$

which coincides with (7.4).

\section{Acknowledgements}

We gratefully acknowledge the support of the NATO grant PST.CLG.980408 and the remarks of a referee, which led to improvements and corrections to the first draft of the paper.

\section{References}

Alili, L., Dufresne, D. And Yor, M. (1997). Sur l'identité de Bougerol pour les fonctionnelles exponentielles du mouvement brownien avec drift. In Exponential Functionals and Principal Values Related to Brownian Motion, Revista Matemática Iberoamericana, Madrid, pp. 3-14.

Comtet, A. ANd Monthus, C. (1996). Diffusion in a one-dimensional random medium and hyperbolic Brownian motion. J. Phys. A 29, 1331-1345.

Gertsenshtein, M. E. And Vasiliev, V. B. (1959). Waveguides with random inhomogeneities and Brownian motion in the Lobachevsky plane. Theory Prob. Appl. 3, 391-398.

Gruet, J. C. (1996). Semi-groupe du mouvement brownien hyperbolique. Stoch. Stoch. Rep. 56, 53-61.

Gruet, J. C. (1998). On the length of the homotopic Brownian word in the thrice punctured sphere. Prob. Theory Relat. Fields 111, 489-516.

Gruet, J. C. (2000). A note on hyperbolic von Mises distributions. Bernoulli 6, 1007-1020.

Karpelevich, F. I., Tutubalin, V. N. And Shur, M. C. (1959). Limit theorems for the composition of distributions in the Lobachevsky plane. Theory Prob. Appl. 3, 399-401.

KolesniK, A. D. (2001). Weak convergence of a planar random evolution to the Wiener process. J. Theoret. Prob. 14, 485-494.

Lalley, S. P. ANd Sellke, T. (1997). Hyperbolic branching Brownian motion. Prob. Theory Relat. Fields 108, 171-192. 
LAO, L. AND Orsingher, E. (2007). Hyperbolic and fractional hyperbolic Brownian motion. To appear in Stochastics. Monthus, C. And Texier, C. (1996). Random walk on the Bethe lattice and hyperbolic Brownian motion. J. Phys. A 29, 2399-2409.

Orsingher, E. (1990). Probability law, flow function, maximum distribution of wave-governed random motions and their connections with Kirchoff's laws. Stoch. Process. Appl. 34, 49-66.

Ratanov, N. (1999). Telegraph evolutions in inhomogeneous media. Markov Process. Relat. Fields 5, 53-68.

Rogers, L. C. G. AND Williams, D. (1987). Diffusions, Markov Processes and Martingales, Vol. 2, Itô Calculus. John Wiley, Chichester.

Royster, D. C. (2004). Non-Euclidean geometry. http://www.math.uncc.edu/ droyster/courses/spring04/index.html. Simon, T. (2002). Concentration of the Brownian bridge on the hyperbolic plane. Ann. Prob. 30, 1977-1989.

Terras, A. (1985). Harmonic Analysis on Symmetric Spaces and Applications. I. Springer, New York. Yor, M. (1992). On some exponential functionals of Brownian motion. Adv. Appl. Prob. 24, 509-531. 